\title{
Performance Analysis of IEEE 802.15.3 MAC Protocol with Different ACK Polices
}

\author{
S. Mehta and K.S. Kwak \\ Wireless Communications Research Center, Inha University, Korea \\ suryanand.megmail.com
}

\begin{abstract}
The wireless personal area network (WPAN) is an emerging wireless technology for future short range indoor and outdoor communication applications. The IEEE 802.15.3 medium access control (MAC) is proposed, specially, for short range high data rates applications, to coordinate the access to the wireless medium among the competing devices. This paper uses analytical model to study the performance analysis of WPAN (IEEE 802.15.3) MAC in terms of throughput, efficient bandwidth utilization, and delay with various acknowledgment schemes under different parameters. Also, some important observations are obtained, which can be very useful to the protocol architectures. Finally, we come up with some important research issues to further investigate the possible improvements in the WPAN MAC.
\end{abstract}

Keywords: Analytical Modeling, Performance Analysis, MAC Protocol, IEEE 802.15.3.

\section{Introduction}

The IEEE standard 802.15.3 MAC layer [1] is based on a centralized, connection oriented topology which divides a large network into several smaller ones termed "piconets". A piconet consists of a Piconet Network Controller (PNC) and DEVs (DEVices). The DEV is designed to be low power and low cost. One DEV is required to perform the role of PNC (Piconet Coordinator), which provides the basic timing for the piconet as well as other piconet management functions, such as power management, Quality of Service (QoS) scheduling, security, and so on. The standard also allows for the formation of child piconets and neighbor piconets. The original piconet is called the parent piconet and the child/neighbor piconets are called the dependent piconets. These piconets differ in the way they associate themselves to the parent piconet. IEEE 802.15.3 standard supports multiple power saving modes and multiple acknowledgement (ACK) policies (NO ACK, Imm-ACK, and Dly-ACK,). It is very robust and supports coexistence with the other WLAN technologies such as IEEE 802.11. In IEEE 802.15.3 MAC protocol, although communications are connectionbased under the control of the PNC, connections and data transfer can be made with peer to peer connections. In IEEE 802.15.3 MAC protocol, the channel time is divided into superframes, which each superframe beginning with a beacon. The superframe is composed of the three major parts: the beacon, the optional contention access 
period (CAP), and the channel time allocation period (CTAP) or channel allocation time (CTA).

Wireless channel is usually vulnerable to errors. Error control mechanisms should be designed in MAC protocol to provide a cretin level of reliability for the higher network layers. In accordance with that, IEEE 802.15.3 defines three types of acknowledgment mechanisms for CTAs: the No-ACK, Imm-ACK, and Dly-ACK mechanisms. For the Imm-ACK and Dly-ACK mechanisms, retransmission is adopted to recover corrupted frames in previous transmissions. For the No-ACK mechanism, ACK is not sent after a reception. In the Imm-ACK mechanism, each frame is individually ACKed following the reception of the frame. The Dly-ACK mechanism allows the source to send multiple frames without waiting for individual ACKs. Instead, the ACKs of the individual frames are grouped into a single response frame to be sent to the source DEV. In [2] and some other literature proposed implied-acknowledgment (Imp-ACK) for bidirectional communication. Implied acknowledgement (Imp-ACK) permits a CTA to be used bi-directionally within a limited scope. Implied ACK policy is not allowed in the CAP to avoid ambiguities between a frame that is transmitted in response to a frame with an implied ACK request and a frame that is transmitted independently when the original frame was missed. To reduce the overhead of the IEEE 802.15.3 MAC, we combine the frame aggregation concept [3] and Dly-ACK mechanism with different burst sizes, and we define this new mechanism as Dly-ACK-AGG. The idea of frame aggregation is to aggregate multiple MAC frames into a single (or approximately single) transmission.

All these ACK policies have a large impact on the throughput, delay, and channel utilization of the network and required a detailed study to find overall performance of the network. In this paper, we present the performance analysis of IEEE 802.15.3 from protocol architecture's point of view. Furthermore, we show the effect of aggregation with Dly-ACK, i.e. Dly-ACK-AGG, on the network performance.

\section{Related Works}

To the best of our knowledge, there is little work on the performance or channel analysis of IEEE 802.15.3 MAC with respect to different ACK policies, under different parameters. However, a large amount of literature is available on IEEE 802.15.3 MAC scheduling, optimization of superframe size, various traffic analyses and so on. Some of the important related works are as follow.

In [4] authors, presents the implementation of IEEE 802.15.3 module in ns-2 and discuses various experimental scenario results including various scheduling techniques. Specially, to investigate the performance of real-time and best-effort traffic with various super frame lengths and different ACK policies. In [5] authors, presents a novel CTA sharing protocol, called VBR-MCTA that enables the sharing of CTAs belonging to streams with the same group identity. This feature allows the proposed protocol to exploit the statistical characteristics of variable bit rate (VBR) streams by giving unused time units to a flow that requires peak rate allocation. And they presents two optimizations to VBR-MCTA, namely VBR-Blind and VBR-TokenBus, as well. In [6, 7], the authors proposed an adaptive Dly-ACK scheme for both TCP and UDP traffic. The first one is to request the Dly-ACK frame adaptively or change the 
burst size of Dly-ACK according to the transmitter queue status. The second is a retransmission counter to enable the destination DEV to deliver the MAC data frames to upper layer timely and orderly. While later is more focused on optimization of channel capacity. Both papers laid a good foundation in simulation and analytical works of IEEE 802.15.3 MAC protocol. Similarly, in [8] authors, formulate a throughput optimization problem under error channel condition and derive a closed form solution for the optimal throughput. The work presented in [8] is close to our work but their analysis scope is limited only in terms of throughput analysis. While our work span covers the delay, throughput, and channel utilization with different ACK policies under frame aggregation and error channel condition.

The paper is outlined as follows. In section 3 we present the performance analysis from protocol architecture's point of view and finally, conclusions and future work are drawn in section 4 .

\section{Performance Analysis of IEEE 802.15.3 MAC Protocol}

In this section, we present the performance analysis of IEEE 802.15.3 MAC to answer several questions like optimization of payload, optimization of ACK policies, effect of aggregation etc., under various parameters.

\subsection{Analytical Model}

We use ground works of Bianchis's model [9] and [8] to present our analysis work. We divide our analysis in two parts; CTA analysis and CAP analysis. Table 1 shows the notations that we used for the analytical model.

Table 1. Parameters notations

$\begin{array}{ll}T_{S I F S} & \text { Short Inter Frame Space (SIFS) time } \\ T_{D I F S} & \begin{array}{l}\text { Distributed Coordinate Function Inter Frame Space (DIFS) } \\ \text { time }\end{array} \\ T_{M I F S} & \text { Minimum Inter Frame Space (MIFS) time } \\ C W_{\text {min }} & \text { Minimum back-off window size } \\ T_{\text {pre. }} & \text { Transmission time of the physical preamble } \\ T_{P H Y} & \text { Transmission time of the PHY header } \\ L_{M A C-H} & \text { MAC overhead in bytes } \\ L_{A C K} & \text { ACK size in bytes } \\ L_{\text {Data }} & \text { Payload size in bytes } \\ T_{M A C-H} & \text { Transmission time of MAC overhead } \\ T_{A C K} & \text { ACK transmission time } \\ T_{\text {Data }} & \text { Transmission time for the payload } \\ T_{f-C A P} & \text { The time for a transmission considered failed during CAP } \\ T_{s-C A P} & \text { The time for a transmission considered successful during CAP } \\ T_{f-C T A} & \text { The time for a transmission considered failed during CAT } \\ T_{s-C T A} & \text { The time for a transmission considered successful during CAT } \\ T_{A C K-T O} & \text { The time-out value waiting for an ACK } \\ T h_{C A P} & \text { Normalized throughput during a CAP time } \\ T h_{C T A} & \text { Normalized throughput during a CTA time } \\ n_{\text {burst }} & \text { Burst size in packets }\end{array}$


The throughput is given by

$$
T h=\frac{\text { Transmitted Data }}{\text { Transmission Cycle Duration }}
$$

We assume a Gaussian wireless channel model. The channel bit error rate (BER), denoted as $p_{e} \quad\left(0<p_{e}<1\right)$, can be calculated via previous frames or some other mechanism. How to obtain $p_{e}$ is way out of the scope of this paper. From [9], a frame with a length $L$ in bits, the probability that the frame is successfully transmitted can be calculated as

$$
p_{s}=\left(1-p_{e}\right)^{L}
$$

Here, for the simplicity we assume that a data frame is considered to be successfully transmitted if both data frame and ACK are successfully transmitted in different ACK mechanism policies. We use Imm_ACK, No_ACK, D_ACK, and D_AGG_ACK to denote the immediate acknowledgement, No acknowledgement, delay acknowledgement, and delay acknowledgement with aggregation, respectively. Then we can define $p_{s}$ for different ACK mechanisms as follows

$$
\begin{aligned}
& p_{s}, \mathrm{Imm} \_\mathrm{ACK}=\left(1-p_{e}\right)^{\left(L_{\text {Data }}+L_{M A C-H}+L_{A C K-I \mathrm{~mm}}\right) * 8} \\
& p_{s}, \mathrm{No} \_\mathrm{ACK}=\left(1-p_{e}\right)^{\left(L_{\text {Data }}+L_{M A C-H}\right) * 8} \\
& p_{s}, \text { Dly_ACK }=\left(1-p_{e}\right)^{\left(L_{D a t a}+L_{M A C-H}+L_{A C K-D l y}\right) * 8} \\
& \left.p_{s}, \text { Dly_ACK-AGG }=\left(1-p_{e}\right)^{\left(L_{D a t a}+L_{M A C-H}+L_{M A C-H s}+L_{A C K-D l y}\right.}\right) * 8
\end{aligned}
$$

A successful transmission time during a CTA is given by ${ }^{1}$

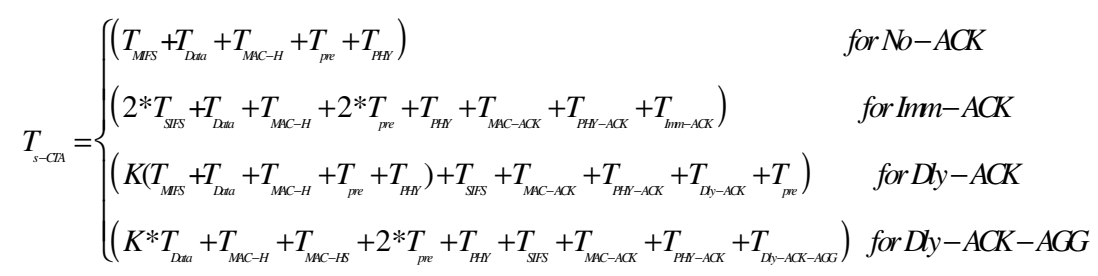

\footnotetext{
${ }^{1}$ Readers are advice to have a look at table 1 while referring equations as we couldn't explain every parameter due to space limitation.
} 
from (2), (3) and (4) the normalized throughput during a CTA is given by

$$
T h_{C T A}= \begin{cases}\frac{p_{s}, N o_{-} A C K L_{\text {Data }} * 8}{T_{s-C T A}} & \text { for No-ACK } \\ \frac{p_{s}, I m m_{-} A C K L_{\text {Data }} * 8}{T_{s-C T A}} & \text { for Imm-ACK } \\ \frac{p_{s}, D l y_{-} A C K K L_{\text {Data }} * 8}{T_{s-C T A}} & \text { for Dly-ACK } \\ \frac{p_{s}, D l y_{-} A C K-A G G K L_{\text {Data }} * 8}{T_{s-C T A}} & \text { for Dly-ACK-AGG }\end{cases}
$$

To demonstrate the effect of n-Dly-ACK and n-Dly-ACK-AGG on bandwidth utilization, we define a metric named maximum effective bandwidth (MEB) based on [10], which is a fraction of time the channel is used to successfully transmit data frames versus the total channel time. The maximum effective bandwidth utilization during a CTA/CAP slot is given by

$$
\begin{gathered}
M E B_{C T A}= \begin{cases}n_{\text {burst }} \cdot \frac{L_{\text {Data }} p_{s}, D l y_{-} A C K}{T_{s-C T A}} & \text { for Dly-ACK } \\
n_{\text {burst }} \cdot \frac{L_{\text {Data }} p_{s}, D l y_{2} A C K-A G G}{T_{s-C T A}} & \text { for Dly-ACK-AGG }\end{cases} \\
M \mathbb{E B}_{C A P}= \begin{cases}n_{\text {bust }} \cdot \frac{L_{\text {Data }} n \psi(1-\psi)^{n-1} p_{s}, \text { Dy_ACK }}{T_{s-C A P}} & \text { for Dly-ACK } \\
n_{\text {bust }} \cdot \frac{L_{\text {Data }} n \psi(1-\psi)^{n-1} p_{s}, D y \_A C K-A C G}{T_{s-C A P}} & \text { for D Dy-ACK-AGG }\end{cases}
\end{gathered}
$$

During the CAP, we use the analytical model similar with CSMA/CA mechanism of IEEE 802.11. Also, we study how to optimize the channel throughput using different ACK policies under error channel condition. When the Imm-ACK mechanism is used in CAP, each node adopts CSMA/CA with binary exponential backoff. When NO-ACK mechanism is used, each node will use a fixed backoff window as it has no knowledge whether or not the transmitted data frame is successfully received. If the Dly-ACK mechanism is used, as long as the backoff timer of a node reaches zero, the node will first send a number $(K)$ of data frames each separated by an MIFS and a delay-request frame separated by an MIFS, and wait for the ACK. If a burst transmission (of $K$ data frames) is considered successful, the sender will reset the backoff window to the initial value; otherwise, the backoff window will be doubled. Dly-ACK-AGG follows the 
same backoff procedure as Dly-ACK. From [9], the failure probability of a transmission during a CAP is given by

$$
p_{f}= \begin{cases}1-(1-p) p_{s}, I m m_{-} A C K & \text { for Imm_ACK } \\ 1-(1-p) p_{s}, D l y_{-} A C K, & \text { forDly_ACK } \\ 1-(1-p) p_{s}, D l y_{-} A C K-A G G, & \text { for Dly_ACK-AGG }\end{cases}
$$

For $n$ number of stations, the probability of a transmitted frame collision is given by

$$
p=1-(1-\psi)^{n-1}
$$

$\psi$, probability of a station to transmit during a generic slot time is also depends on number of retry limit. Then, the probability of the busy channel is given by

$$
p_{b}=1-(1-\psi)^{n}
$$

From (8) and (9), the probability of a success transmission occurs in a slot time is given by

$$
p_{S}= \begin{cases}n \psi(1-\psi)^{n-1} p_{s}, N o_{-} A C K, & \text { for } N o-A C K \\ n \psi(1-\psi)^{n-1} p_{s}, \operatorname{Im}{ }_{2} A C K, & \text { for Imm } \operatorname{Im}_{-} A C K \\ n \psi(1-\psi)^{n-1} p_{s}, D y_{-} A C K, & \text { for } D y_{-} A C K \\ n \psi(1-\psi)^{n-1} p_{s}, D y_{-} A C K-A G G, A G G\end{cases}
$$

A successful transmission time during a CAP is given by

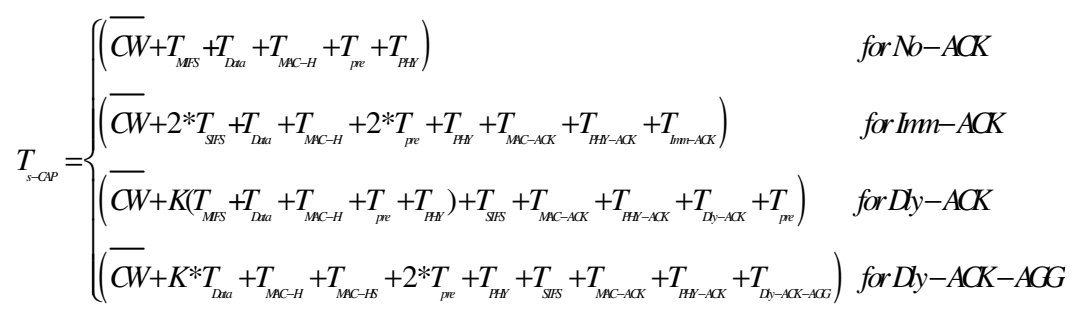

where $\overline{C W}$ represents the average back-off time. The average back-off defines the back-off duration for "light loaded networks", i.e. when each station has access to the channel after the first back-off attempt and is given by

$$
\overline{C W}=\frac{C W_{\min } \cdot T_{s l o t}}{2}
$$


A failure transmission time during a CTA is given by

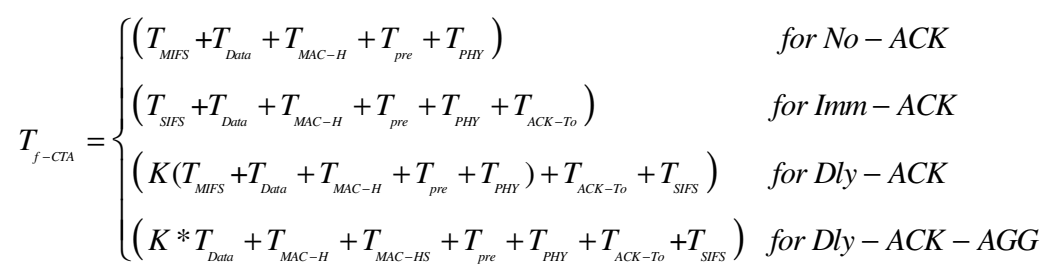

from (10), (11), and (13), the normalized throughput during a CAP is given by

$$
T h_{C A P}= \begin{cases}\frac{P_{S} L_{\text {Data }} * 8}{\left(1-p_{b}\right) \delta+P_{S} T_{s-C A P}+\left(p_{b}-P_{S}\right) T_{f-C A P}} & \text { for No-ACK } \\ \frac{P_{S} L_{\text {Data }} * 8}{\left(1-p_{b}\right) \delta+P_{S} T_{s-C A P}+\left(p_{b}-P_{S}\right) T_{f-C A P}} & \text { for No-ACK } \\ \frac{P_{S} K L_{\text {Data }} * 8}{\left(1-p_{b}\right) \delta+P_{S} T_{s-C A P}+\left(p_{b}-P_{S}\right) T_{f-C A P}} & \text { for Dly-ACK } \\ \frac{P_{S} K L_{\text {Data }} * 8}{\left(1-p_{b}\right) \delta+P_{S} T_{s-C A P}+\left(p_{b}-P_{S}\right) T_{f-C A P}} & \text { for Dly-ACK-AGG }\end{cases}
$$

from (1), we can also calculate the average access delay during a CTA/CAP.

\subsection{Performance Evaluation}

For the performance evaluation, we adopt the following parameters from [11] as shown in table 2 .

Table 2. Parameters values

\begin{tabular}{|c|c|}
\hline Parameters & Values \\
\hline SIFS & 2.5 usec \\
\hline MIFS & 1 usec \\
\hline Preamble and PLCP Header & 9 usec \\
\hline$C W_{\text {min }}$ & 8 \\
\hline Payload Size & $1 \sim 5 \mathrm{~KB}$ \\
\hline ACK Policy & 3 basic +Dly-ACK-AGG policies \\
\hline Data Rate & $1 \sim 2$ Gbps \\
\hline Control Signal Rate & 48 Mbps \\
\hline
\end{tabular}

\subsubsection{CTA Analysis}

Figures 1 and 2 show the normalized throughput for different payload sizes with different ACK polices with and without aggregation, respectively. We assume an ideal channel conditions for these results. Here, we can observe that No-ACK gives the 
superior results as most of the CTA time is utilized for data transfer. However, NoACK policy is not suitable for every application and channel condition. The Dly-ACKAGG policy can achieve somewhat close results to No-ACK policy, as it reduces the unnecessary inter-frame time as well as the header size. Figures 1 and 2 give us the normalized value of throughput at different payload sizes but can't answer for optimum payload size. So, we produce the same results with Gaussian wireless channel model with a given BER rate.

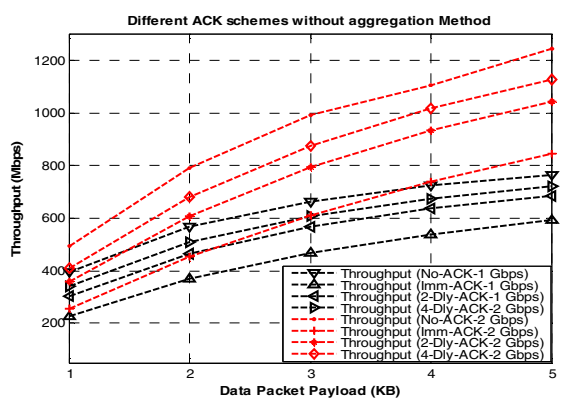

Fig. 1. Throughput versus payload size with different ACK policies

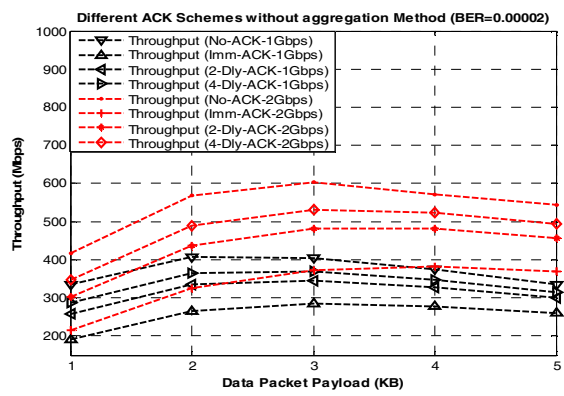

Fig. 3. Throughput versus payload size with different ACK policies

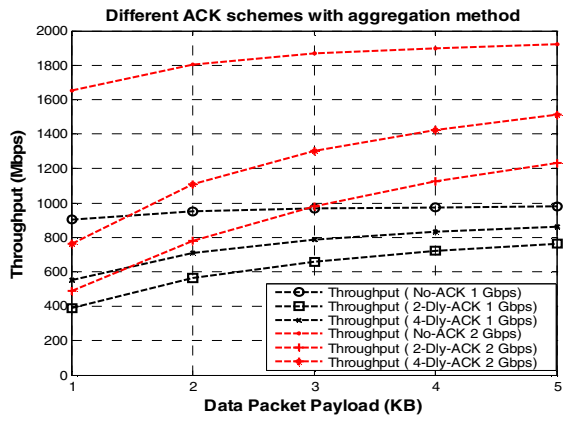

Fig. 2. Throughput versus payload size with different ACK policies

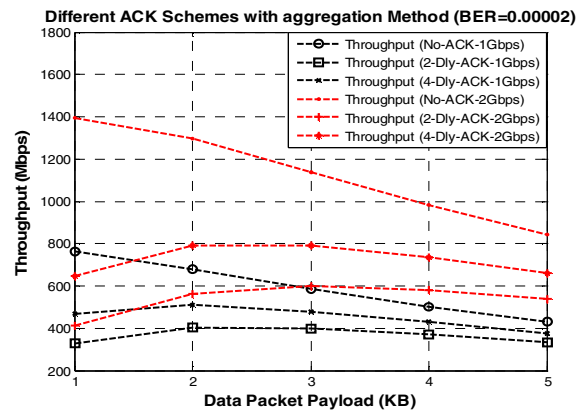

Fig. 4. Throughput versus payload size with different ACK policies

Figures 3 and 4 show the throughput for different payload sizes under a given BER value. It can be seen that an optimal payload size exists for a given BER value. As shown in the mentioned figures the throughput first increases, and then decreases with increasing payload size (even with the aggregation) in error prone channels. This is because without the protection of FCS in individual payload frame, a single bit error may corrupt the whole frame which will waste lots of medium time usage and counteract the efficiency produced by an increased payload size. Figure 5 shows the normalized throughput for different BER values with different ACK policies when payload size is set to $1 \mathrm{~KB}$. As the BER value increases the throughput decreases. The No-ACK 
policy with aggregation has larger throughput over large range of BER values than any other ACK policies. To find the effect of n-Dly-ACK on bandwidth utilization as well as to find the optimal value of burst size for n-Dly-ACK policy, we define the MEB metric in (6). Figures 6 and 7 show the MEB with different burst values under a given $\mathrm{BER}$ value. From these figure we can observe that burst size $=4$ gives good results in fairly all given payload values. Figure 8 shows the access delay performance for different burst sizes with the aggregation method. Here, we only analyzed n-Dly-ACKAGG policy to get the upper bound on the delay performance.

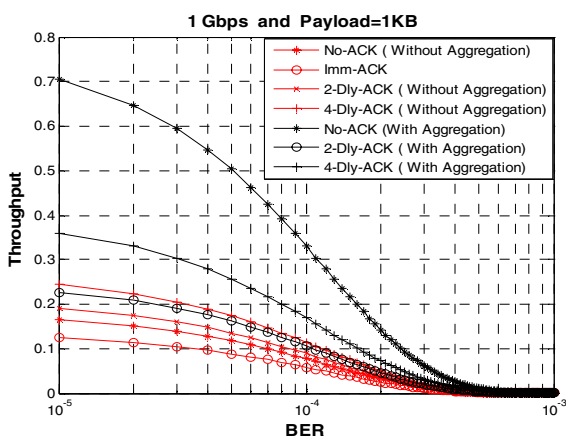

Fig. 5. Throughput versus BER value with different ACK policies

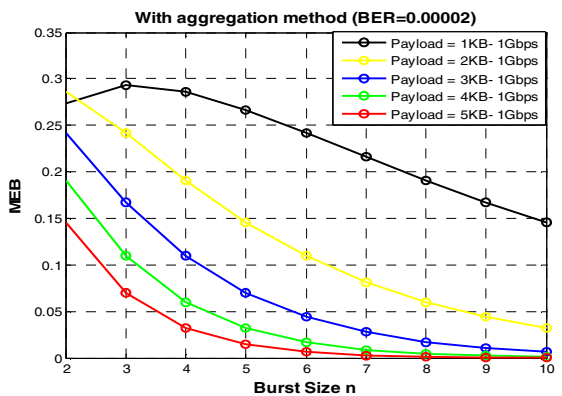

Fig. 7. MEB versus burst size

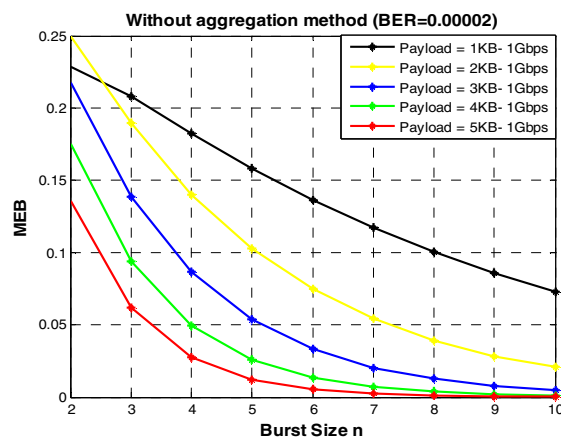

Fig. 6. MEB versus burst size

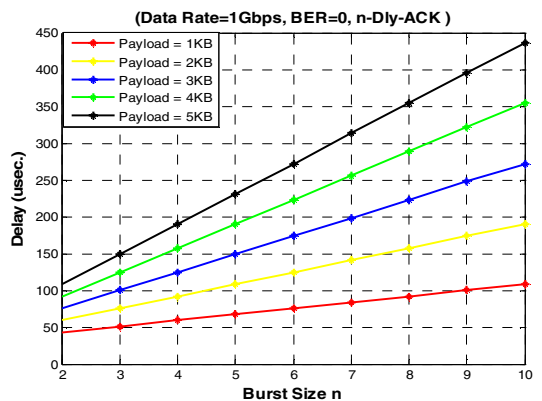

Fig. 8. Access delay versus burst size

\subsubsection{CAP Analysis}

Figures 9 and 10 show the normalized throughput over different payload sizes with different ACK policies. Here, we assume no competition between active nodes, as our main focus is to get maximum normalized throughput for each payload size. Figures 11 and 12 show the normalized throughput for different payload sizes with different ACK policies under a given BER value. For each ACK policies, with the increase of the payload size, the throughput first increases, then decreases after the maximal point. This can be explained as follows. In CAP, the time to transmit the payload is only a 
small portion of the total time used. Therefore, when the payload size increases, the transmission efficiency can be increase, but the error probability also increases. The increase of the curves in figures 11 and 12 is because the effect of increased transmission efficiency is more significant than the effect of increased frame error probability, and the decrease of the curves is due to dominant effect of increased frame error probability when payload size further increases. From the above mentioned figures we can find out the optimum payload size value for a given BER value. Here, it is worth to note that normalized throughput performance depends on the number of active stations and backoff window size during a CAP time. Figure 13 shows the normalized throughput for different BER values when payload size is set to $1 \mathrm{~KB}$.

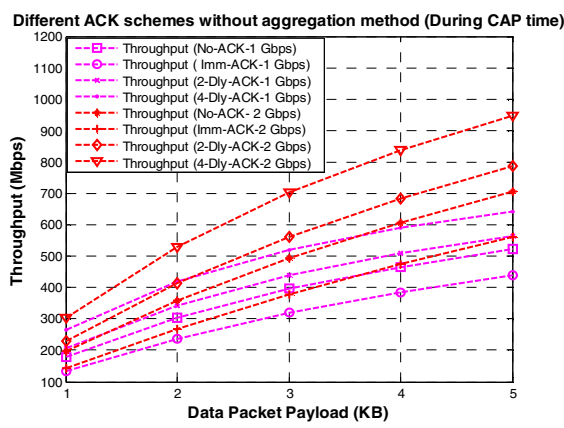

Fig. 9. Throughput versus payload size

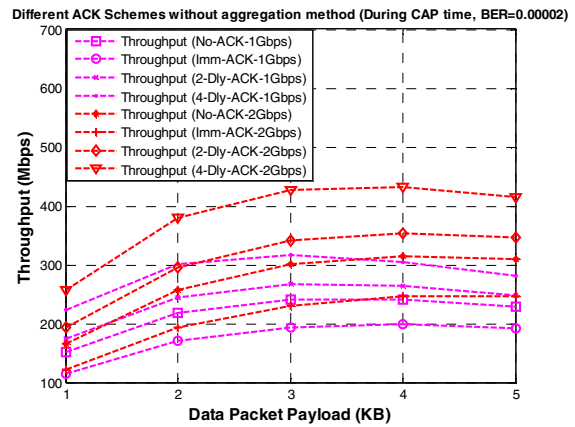

Fig. 11. Throughput versus payload size

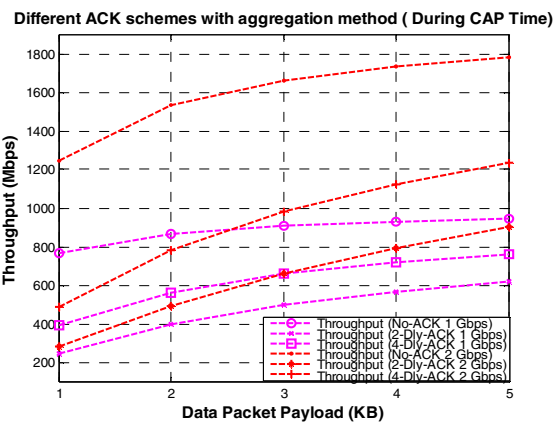

Fig. 10. Throughput versus payload size

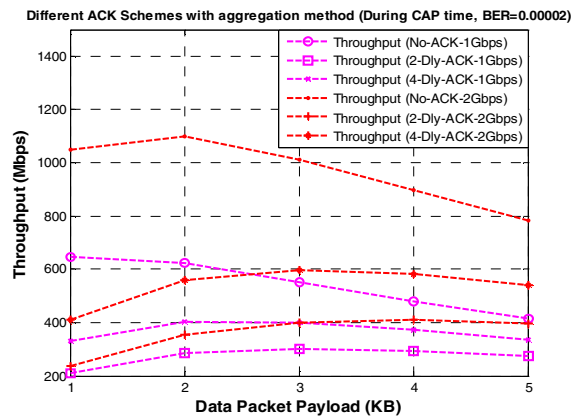

Fig. 12. Throughput versus payload size

Similar to CTA duration analysis, Figure 14 and 15 shows the MEB for different burst values under a given BER value. Figure 16 shows the access delay performance for different burst sizes under a given BER value. The access delay performance with different ACK policies is very sensitive to backoff window size and number of active nodes. In this paper, we focused only on n-Dly-ACK-AGG policy to get the upper bound on the delay performance. 


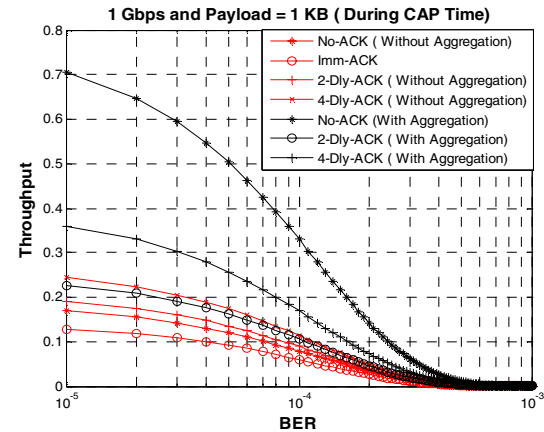

Fig. 13. Throughput versus BER value with different ACK policies

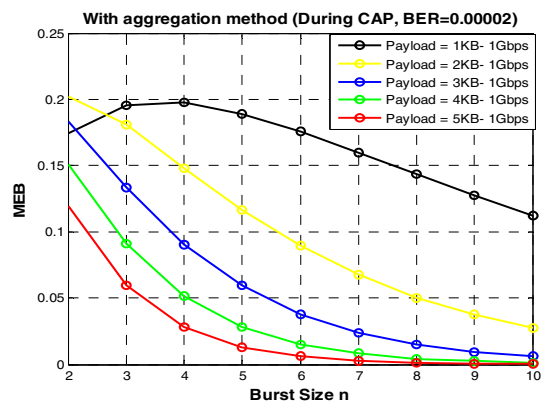

Fig. 15. MEB versus burst size

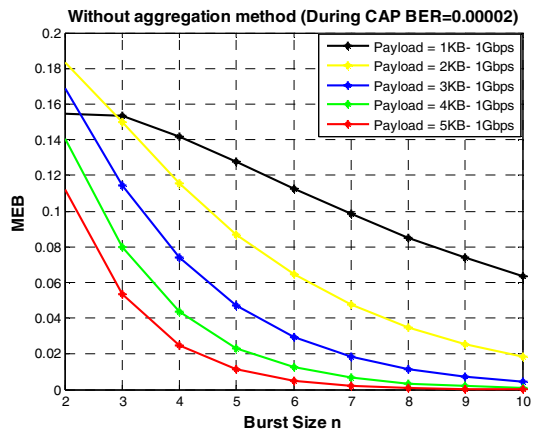

Fig. 14. MEB versus burst size

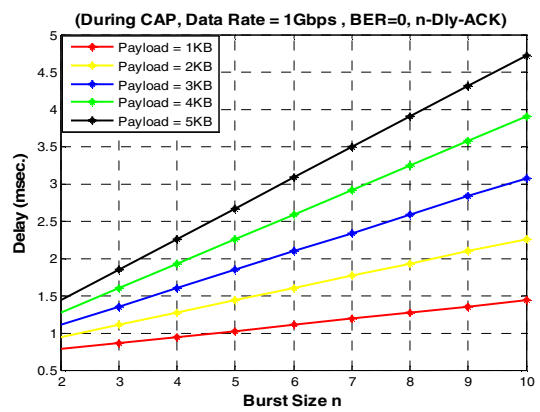

Fig. 16. Access delay versus burst size

\section{Conclusions and Future Work}

In this paper, we presented a performance analysis of WPAN (IEEE 802.15.3) MAC for wireless sensor networks. We have extensively studied the different ACK policies in CTA and CAP under a given BER value. The optimal payload size as well as optimal burst size can be determined analytically from the presented analysis. For future work, we want to study the impact of backoff window size on channel utilization and access delay. We hope that the results of this paper will help sensor network designers to easily and correctly provision systems based on IEEE 802.15.3 MAC technology.

\section{References}

1. P802.15.3/D17: (C/LM) Standard for Telecommunications and Information Exchange Between Systems - LAN/MAN Specific Requirements - Part 15.3: Wireless Medium Access Control (MAC) and Physical Layer (PHY) Specifications for High Rate Wireless Personal Area Networks (February 2003) 
2. Part 15.3: Wireless Medium Access Control (MAC) and Physical Layer (PHY) Specifications for High Rate Wireless Personal Area Networks. Amendment 1: MAC Sub layer, IEEE Std 802.15.3b (February 2005)

3. Xiao, Y.: IEEE 802.11n: Enhancements for higher throughput in wireless LANs. IEEE Wireless Communications, 82-91 (December 2005)

4. Chin, K.-W., Lowe, D.: Simulation study of the IEEE 802.15.3 MAC. In: Proc., Australian Telecommunications and Network Applications Conference (ATNAC), Sydney, Australia (2004)

5. Chin, K.W., Lowe, D.: A Novel IEEE 802.15.3 CTA Sharing Protocol for Supporting VBR Streams. In: Proc. of IEEE ICCCN (2005)

6. Chen, H., Guo, Z., Yao, R., Li, Y.: Improved Performance with Adaptive Dly-ACK for IEEE 802.15.3 WPAN over UWB PHY. IEICE Trans. Fundamentals E88-A(9) (September 2005)

7. Tseng, Y.H., Wu, E.H., Chen, G.H.: Maximum Traffic Scheduling and Capacity Analysis for IEEE 802.15.3 High Data Rate MAC Protocol. In: Proc. of IEEE VTC (2003)

8. Xiao, Y., Shen, X., Jiang, H.: Optimal ACK Mechanisms of the IEEE 802.15.3 MAC for Ultra-Wideband Systems. IEEE JSAC 24(4) (April 2006)

9. Bianchi, G.: Performance analysis of the IEEE 802.11 distributed coordination function. IEEE JSAC 18(3), 535-547 (2000)

10. Chen, H., Guo, Z., Yao, R.Y., Shen, X., Li, Y.: Perfoamnce Analyis of delyed Acknowledgemnet Scheme in UWB-Based High-Rate WPAN. IEEE transaction on vehicular technology 55(2) (March 2006)

11. IEEE P802.15 Working Group for Wireless Personal Area Networks (WPANs).: Unified and flexible millimeter wave WPAN systems supported by common mode. DOC: IEEE 802.15-07-0761-05-003c (July 2009) 